Classification

Physics Abstracts

$61.10 \mathrm{D}-61.12 \mathrm{~B}-61.30$

\title{
Dynamics of the undulation mode in swollen lamellar phases
}

\author{
René Messager, Patricia Bassereau and Grégoire Porte \\ Groupe de Dynamique des Phases Condensées, USTL, CC 26, 34095 Montpellier Cedex 5, \\ France
}

(Reçu le 17 novembre 1989, accepté le 6 mars 1990)

\begin{abstract}
Résumé. - Nous étudions la dynamique du mode d'ondulation (vecteur d'onde de déplacement q parallèle aux couches) de phases lamellaires diluées. Nous calculons l'équation de dispersion de ce mode pour tous les vecteurs d'onde, i.e. des petits aux grands $q$ (par rapport à l'inverse de la distance entre couches). Nous calculons ensuite le facteur de structure statique pour des vecteurs de diffusion $\mathbf{Q}$ parallèles aux couches sur toute la gamme de $Q$, ainsi que le facteur de structure dynamique aux grands $Q$.
\end{abstract}

\begin{abstract}
We investigate the dynamics of the undulation mode (displacement of wave vector $q$ parallel to the layers) in swollen lamellar phases. We calculate the dispersion equation of this mode for all wave vectors, from small $q$ to high $q$ (compared with the inverse of the layer spacing). We then calculate the static structure factor for scattering wave vectors $\mathbf{Q}$ parallel to the layers over the whole $Q$-range and also the dynamic structure factor at high $Q$.
\end{abstract}

\section{Introduction.}

Some amphiphilic solutions in water or oil (or possibly both) may give lamellar phases, i.e. solutions of periodically stacked bilayers [1], under certain conditions (concentration, temperature, salinity, presence of a cosurfactant...).

These mixtures present typical defects (focal conic textures) which are characteristic of a quasi-long range smectic order. They can often be swollen by addition of solvent [2], and the smectic period $(d)$ then becomes much larger than the thickness $(e)$ of the bilayers $(d \gg e)$.

The structure and stability of these swollen phases are now well understood. Their properties are basically controlled by $k_{\mathrm{c}}$, the rigidity modulus of the bilayers, and by the steric interaction induced by the thermal undulations of the layers. For large scale displacement fluctuations $(\gg d)$, the steric interaction essentially acts like an harmonic interaction characterized by an equivalent smectic compressibility modulus $\overline{\mathbf{B}}_{\text {st }}$ [3], and the static structure factor as measured by X-ray or neutron scattering is therefore well understood in the vicinity of the Bragg peak $Q_{\mathrm{B}}$ [4] and also at very small $Q$ when considering concentration fluctuations associated with the fluctuations of the interlayer spacing [5].

Still in the low $q$ limit, the hydrodynamics of all deformation modes of such binary smectics has been investigated in detail [6], allowing a complete quantitative treatment of dynamic light scattering data. 
In this paper, we focus on displacement modes having a wave vector $\mathbf{q}$ parallel to the layers $\left(q_{z}=0, q_{\perp}=q\right)$. We use a local formalism to investigate the hydrodynamics of such modes and so obtain the dispersion relation for all values of $q_{\perp}$. We then calculate the static and dynamic structure factors $S\left(Q_{\perp}\right)$ and $F\left(Q_{\perp}, t\right)$ for scattering wave vectors $\mathbf{Q}$ parallel to the layers $\left(Q_{z}=0, Q_{\perp}=Q\right)$ as could be measured by X-ray or neutron scattering using well oriented mono-cristalline samples. In opposition to light, these radiations are not sensitive to the local anisotropy of the bilayers. Their interaction with pure undulations are therefore non linear through the concentration fluctuations associated with the local tilt of the bilayers. In spite of the complications brought in by this non-linearity, the static structure factor can be calculated at all $Q$, but the dynamic structure factor is obtained in the high $Q$ limit only.

\section{Hydrodynamics.}

We denote the average spacing between the bilayers by $d$. The $x, y$ plane is the plane of the bilayers. The significant displacement fluctuations are in the $z$ direction and are characterized by the displacement function $u_{n}(x, y)$ for the $n$-th layer (see Fig. 1). The origin of the coordinates $(x, y, z)$ is on the 0 th lamella.

In this section, we calculate the hydrodynamic relaxation time of a sinusoidal displacement mode of the wave vector $\mathbf{q}$ parallel to the layer planes $\left(q_{z}=0, q=q_{\perp}=q_{x}\right)$. The medium is assumed to be uncompressible and isothermal (i.e. the high frequency sound waves and the thermal effects are ignored). This is reasonable since sound waves have sufficiently high frequencies to relax faster than undulations and then not to couple with them (see reference 6 for details), and since temperature and undulation fluctuations are statistically independent [7].

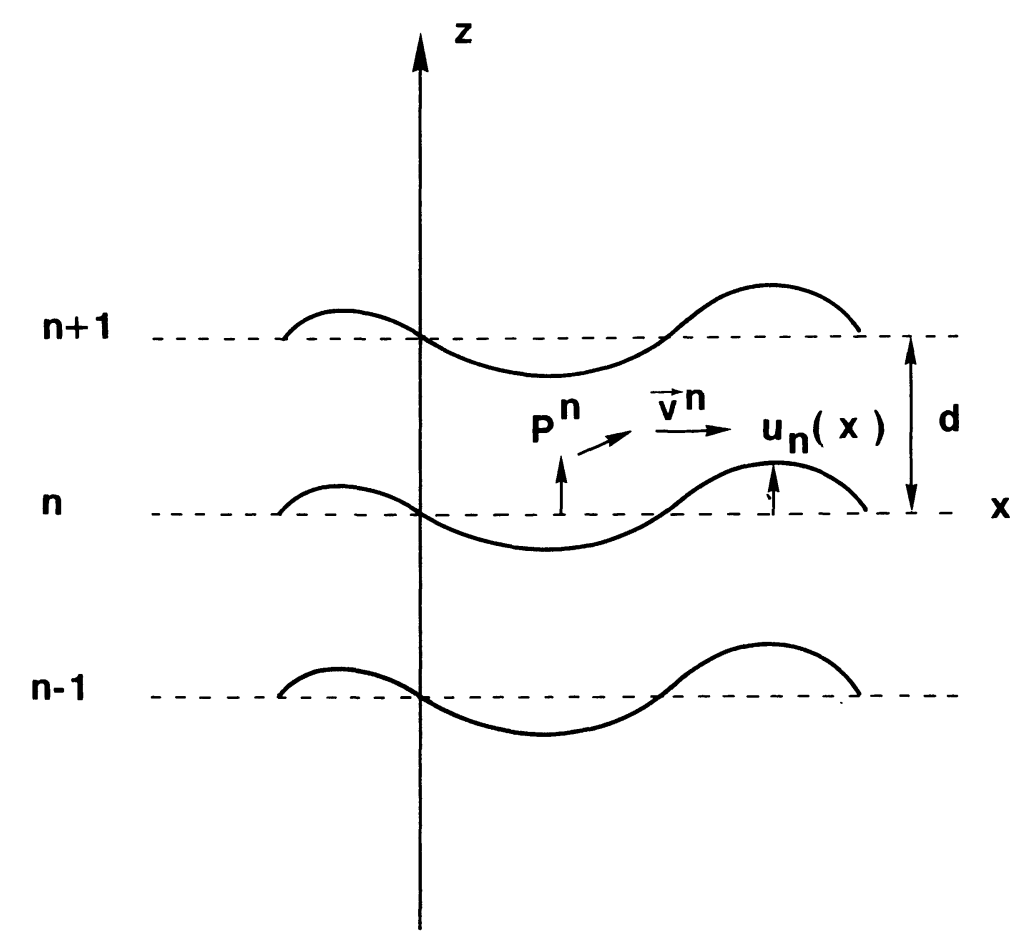

Fig. 1. - Representation of the undulation mode and definitions of local fields. 
The local displacement of the' $n$-th lamella is therefore :

$$
u_{n}(x, t)=u_{0} \exp (i q x-\omega(q) t)
$$

$\omega^{-1}$ is the $q$-dependent relaxation time of this deformation mode. The linearized hydrodynamics equations are :

$$
\begin{gathered}
\operatorname{div}(\mathbf{v})=0 \\
\frac{\partial \mathbf{v}}{\partial t}=-\frac{1}{\rho} \operatorname{grad} P+\nu \Delta \mathbf{v}
\end{gathered}
$$

where $\mathbf{v}$ is the local velocity of the solvent, $\rho$ and $\nu$ are the density and the kinematic viscosity of the solvent, and $P$ is the local pressure of the solvent.

The boundary conditions are, assuming that the lamellae have no thickness (i.e. the solution is dilute enough) and that the displacement amplitude is small in comparison with the wave length :

$$
\begin{aligned}
& v_{x}^{n}(z=n d)=0 \\
& v_{x}^{n}(z=(n+1) d)=0 \\
& v_{z}^{n}(z=n d)=\frac{\mathrm{d} u_{n}}{\mathrm{~d} t} \\
& v_{z}^{n}(z=(n+1) d)=\frac{\mathrm{d} u_{n+1}}{\mathrm{~d} t}
\end{aligned}
$$

with $\mathbf{v}^{n}$ the velocity field between the $n$-th and the $(n+1)$-th lamellae.

$k_{\mathrm{c}}$ being the rigidity modulus of the lamella, the elastic energy density is (8):

$$
\frac{1}{2} k_{\mathrm{c}}\left(\left(\frac{\partial^{2} u_{n}}{\partial x^{2}}\right)+\left(\frac{\partial^{2} u_{n}}{\partial y^{2}}\right)\right)^{2}=\frac{1}{2} k_{\mathrm{c}} q^{4} u_{n}^{2} .
$$

Then, $\sigma$ being the mass of the layer per unit area and $P_{n}$ the pressure field between the $n$-th and the $(n+1)$-th lamellae, the motion equation of the $n$-th lamella is :

$$
\sigma \frac{\mathrm{d}^{2} u_{n}}{\mathrm{~d} t^{2}}=-P_{n}(z=n d)+P_{n-1}(z=n d)-k_{\mathrm{c}} q^{4} u_{n} .
$$

Using equation (1), (8) becomes:

$$
\left(\sigma \omega^{2}+k_{\mathrm{c}} q^{4}\right) u_{n}=-P_{n}(z=n d)+P_{n-1}(z=n d) .
$$

Equations (2) and (3) give $\Delta P_{n}=0$.

We look for solutions of this equation having the form : $P_{n}=f_{n}(z) \exp (i q x-\omega t)$, this , form being chosen according to the boundary conditions.

The solution is easily obtained as :

$$
P_{n}=\left(a_{n} \mathrm{e}^{q z}+b_{n} \mathrm{e}^{-q z}\right) \mathrm{e}^{i q x-\omega t}
$$

where $a_{n}$ and $b_{n}$ are unknonws.

For a pure undulation mode, $P_{n}$ must be periodic in the $z$ direction. We then must have $a_{n}=A \mathrm{e}^{-q n d}$ and $b_{n}=B \mathrm{e}^{q n d}$, where $A$ and $B$ are constants. 
Using (3), we have :

$$
\begin{aligned}
& v_{z}^{n}=\frac{q}{\omega \rho}\left(A \mathrm{e}^{q(z-n d)}-B \mathrm{e}^{-q(z-n d)}+c_{n} \mathrm{e}^{\sqrt{q^{2}-\frac{\omega}{\nu}} z}+d_{n} \mathrm{e}^{-\sqrt{q^{2}-\frac{\omega}{\nu}} z}\right) \mathrm{e}^{i q x-\omega t} \\
& v_{x}^{n}=\frac{i}{\omega \rho} \times \\
& \left(q A \mathrm{e}^{q(z-n d)}+q B \mathrm{e}^{-q(z-n d)}+\sqrt{q^{2}-\frac{\omega}{\nu}} c_{n} \mathrm{e}^{\sqrt{q^{2}-\frac{\omega}{\nu}} z}-\sqrt{q^{2}-\frac{\omega}{\nu}} d_{n} \mathrm{e}^{-\sqrt{q^{2}-\frac{\omega}{\nu}} z}\right) \mathrm{e}^{i q x-\omega t} .
\end{aligned}
$$

For the same reason as above, we must have :

$$
c_{n}=C \mathrm{e}^{-\sqrt{q^{2}-\frac{\omega}{\nu}} n d} \text { and } d_{n}=D \mathrm{e}^{\sqrt{q^{2}-\frac{\omega}{\nu}} n d} \text {. }
$$

Equations (4) to (7) and (9) are thus rewritten :

$$
\left\{\begin{array}{c}
A+B+V C-V D=0 \\
\mathrm{e}^{q d} A+\mathrm{e}^{-q d} B+V \mathrm{e}^{V q d} C-V \mathrm{e}^{-V q d} D=0 \\
A-B+C+D+E=0 \\
A \mathrm{e}^{q d}-B \mathrm{e}^{-q d}+C \mathrm{e}^{V q d}+D \mathrm{e}^{-V q d}+E=0 \\
A\left(\mathrm{e}^{q d}-1\right)+B\left(\mathrm{e}^{-q d}-1\right)+\alpha E=0
\end{array}\right.
$$$$
\text { with }\left\{\begin{aligned}
E & =\frac{\rho \omega^{2}}{q} u_{0} \\
x & =\frac{\omega}{\nu q^{2}} \\
V & =\sqrt{1-x} \\
\alpha & =-\left(\sigma \omega^{2}+k_{\mathrm{c}} q^{4}\right) \frac{q}{\rho \omega^{2}}
\end{aligned}\right.
$$

We now have 5 equations and 5 unknowns. The problem is then solved. The system being homogeneous, its determinant must be zero. This condition will give us the relaxation frequency $\omega$.

The determinant $F$ is obtained, after a few manipulation :

$$
\begin{aligned}
& \frac{F}{4}=2 \sqrt{1-x}\{\operatorname{sh}(q d) \cdot[\operatorname{ch}(\sqrt{1-x} q d)-1]+\sqrt{1-x} \operatorname{sh}(\sqrt{1-x} q d) \cdot[1-\operatorname{ch}(q d)]\}+ \\
&+\alpha\{(2-x) \operatorname{sh}(q d) \operatorname{sh}(\sqrt{1-x} q d)+2 \sqrt{1-x}[1-\operatorname{ch}(\sqrt{1-x} q d) . \operatorname{ch}(q d)]\}
\end{aligned}
$$

and $x$ is given by $F=0$, which is necessary to discard the trivial solution $A=B=\cdots=E=0$.

The first solution $x=1$ is a high-frequency shear wave and does not concern us.

The other interesting solution corresponds to $x \ll 1$. So we expand the equation to the first order in $x$, remembering that

$$
\alpha=-\frac{\delta}{x^{2}} \quad \text { with } \quad \delta=\frac{\sigma \omega^{2}+k_{\mathrm{c}} q^{4}}{\rho \nu^{2} q^{3}}
$$

We obtain :

$$
\frac{F}{4}=x[\operatorname{sh}(q d)-q d][\operatorname{ch}(q d)-1]-\frac{\delta}{4}\left[\left(\operatorname{sh}(q d)^{2}-(q d)^{2}\right)\right] .
$$

Then :

$$
x=\frac{\delta}{4} \frac{\operatorname{sh}(q d)+q d}{\operatorname{ch}(q d)-1} .
$$


Finally, the dispersion relation is, assuming that $\sigma \omega^{2} \ll k_{\mathrm{c}} q^{4}$ :

$$
\omega=\frac{k_{\mathrm{c}} q^{3}}{4 \eta} \frac{\operatorname{sh}(q d)+q d}{\operatorname{ch}(q d)-1} .
$$

Typical values are $\eta \approx 10^{-3}$ S.I. and $k_{\mathrm{c}} \approx k_{\mathrm{B}} T$. Then, for a light scattering experiment ( $q \approx 10^{6}$ to $10^{7} \mathrm{~m}^{-1}$ ), the value of $\omega$ will be between $10^{3}$ and $10^{6} \mathrm{~s}^{-1}$, which is indeed measurable. For inelastic neutron scattering (spin-echo) experiment $\left(q \approx 10^{9} \mathrm{~m}^{-1}\right)$. we expect $\omega$ to be about $10^{9} \mathrm{~s}^{-1}$. In both cases one easily checks that $x \leq 10^{-3}$ and that $\sigma \omega^{2} \ll k_{\mathrm{c}} q^{4}$ which is consistent with our assumption.

Let us remark that $\omega$ is real, which means that the system relaxes without oscillations. This is consistent with the fact that the inertial term $\sigma \omega^{2}$ has been neglected.

Let us now focus on the limiting cases:

The limit $q d \ll 1$ is :

$$
\omega=\frac{k_{\mathrm{c}} q^{2}}{\eta d}
$$

consistently with the low- $q$ prediction in reference [6]

The limit $q d \gg 1$ is :

$$
\omega=\frac{k_{\mathrm{c}} q^{3}}{4 \eta}
$$

$\omega$ is independent of $d$, which is consistent with the fact that the high $q$ limit corresponds to local (free-lemalla) behaviour $[9,10]$.

\section{Static X-ray/neutron scattering.}

We here calculate the neutron or X-ray intensity scattered in the direction of the layers. These radiations are only sensitive to local concentration fluctuations (and not to the local anisotropy of the bilayers). Then [11] :

$$
I(\mathbf{Q})=\left\langle\left|\int \delta c(\mathbf{r}) \mathrm{e}^{i \mathbf{Q} \cdot \mathbf{r}} \mathrm{d} \mathbf{r}\right|^{2}\right\rangle
$$

where $c$ is the local concentration and the brackets indicate thermal average.

Since $Q_{z}=0$, to integrate over $z$ simply corresponds to project the lamella onto the $(x, y)$ plane :

$$
\int_{-\infty}^{+\infty} \delta c(\mathbf{r}) \mathrm{d} z=\sum_{n} \int_{-\varepsilon}^{+\varepsilon} \delta c_{n}(\mathbf{r}) \mathrm{d} z
$$

where the integration runs over the thickness $e$ of each bilayer. So finally:

$$
\int_{-\varepsilon}^{+\varepsilon} \delta c_{n}(\mathbf{r}) \mathrm{d} z=2 \varepsilon c_{0}=\frac{e c_{0}}{\cos \left(\theta_{n}(\mathbf{r})\right)}
$$

where $c_{0}$ is the local concentration and $\theta$ the tilt angle between the local director and the $z$ direction (see Fig. 2). $c_{0}$ is related to the interlayer spacing through $c_{0}=2 d^{-1} a^{-2}$ where 


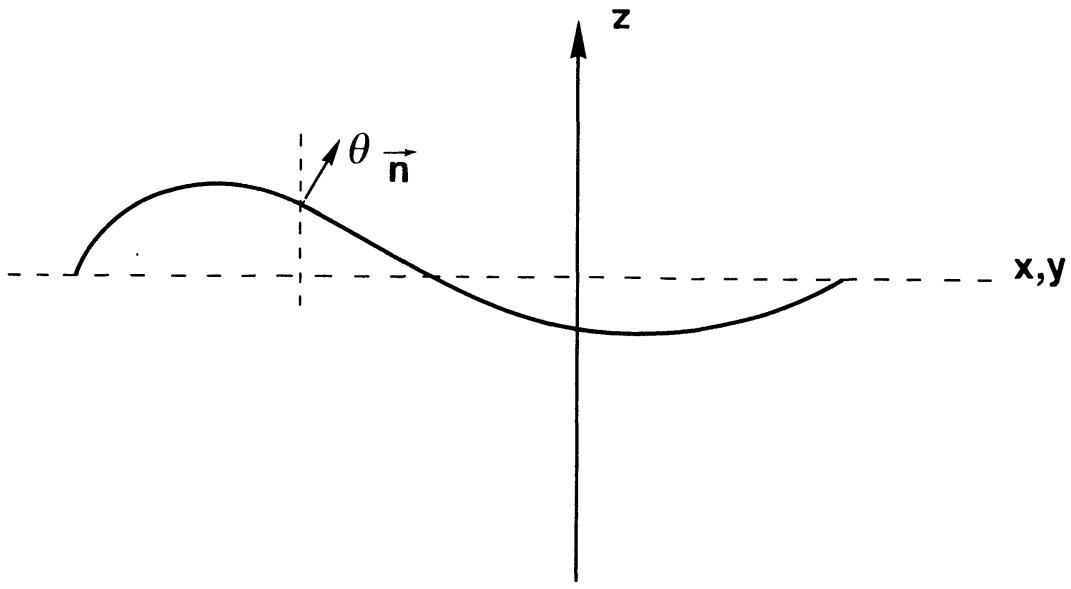

Fig. 2. - Director and $\theta$ angle of the lamella.

$a^{2}$ is the area per polar head. We shall keep for simplicity the integral notation instead of discrete summation over the lamellae.

The director is :

$$
\mathbf{n}=\frac{1}{\sqrt{1+\left(\frac{\partial u}{\partial x}\right)^{2}+\left(\frac{\partial u}{\partial y}\right)^{2}}} \mid \begin{array}{r}
-\frac{\partial u}{\partial x} \\
-\frac{\partial u}{\partial y} \\
1
\end{array}
$$

where $u(x, y)$ is the local displacement of the lamella. We have :

$$
\cos (\theta)=n_{z}=\frac{1}{\sqrt{1+\left(\frac{\partial u}{\partial x}\right)^{2}+\left(\frac{\partial u}{\partial y}\right)^{2}}} \Rightarrow \frac{1}{\cos (\theta)} \cong 1+\frac{1}{2}\left[\left(\frac{\partial u}{\partial x}\right)^{2}+\left(\frac{\partial u}{\partial y}\right)^{2}\right]
$$

in the case of small amplitude fluctuations compared to wave length, which is always verified [8]. The constant term gives a $\delta$ function; we shall forget it :

$$
\Rightarrow I(\mathbf{Q})=\frac{c_{0}^{2}}{4}\left\langle\left.\iint\left[\left(\frac{\partial u}{\partial x}\right)^{2}+\left(\frac{\partial u}{\partial y}\right)^{2}\right] \mathrm{e}^{i \mathbf{Q} \cdot \mathbf{r}} \mathrm{d} \mathbf{r}\right|^{2}\right\rangle
$$

we use the Fourier transform :

$$
u=\sum_{\mathbf{q}} u_{\mathbf{q}} \mathrm{e}^{i \mathbf{q} \cdot \mathbf{r}} \text { with } u_{\mathbf{q}}^{*}=u_{-\mathbf{q}}
$$

then :

$$
\left(\frac{\partial u}{\partial x}\right)^{2}+\left(\frac{\partial u}{\partial y}\right)^{2}=-\sum_{\mathbf{q}, \mathbf{q}_{1}} u_{\mathbf{q}} u_{\mathbf{q}_{1}} \mathrm{e}^{i\left(\mathbf{q}+\mathbf{q}_{1}\right) \cdot \mathbf{r}} \mathbf{q}_{\perp} \cdot \mathbf{q}_{1 \perp}
$$


The index $\perp$ means a projection of $\mathbf{q}$ on the $q_{x}, q_{y}$ plane.

$$
\left.I(\mathbf{Q})=\frac{c_{0}^{2}}{4} \int\left|\sum_{\mathbf{q}, \mathbf{q}_{1}} u_{\mathbf{q}} u_{\mathbf{q}_{1}}\left(\int \mathrm{e}^{i\left(\mathbf{q}+\mathbf{q}_{1}\right) \cdot \mathbf{r}} \mathrm{e}^{i \mathbf{Q} \cdot \mathbf{r}} \mathrm{d} \mathbf{r}\right) \mathbf{q}_{\perp} \mathbf{q}_{1 \perp}\right|^{2}\right\rangle .
$$

The integration gives $V \boldsymbol{\delta}\left(\mathbf{q}+\mathbf{q}_{1}+\mathbf{Q}\right) \Rightarrow \mathbf{q}_{1}=-\mathbf{q}-\mathbf{Q}$ ( $V$ is the volume of the sample). Then :

$$
I(\mathbf{Q})=\frac{V^{2} c_{0}^{2}}{4} \sum_{\mathbf{q}, \mathbf{q}_{2}}\left\langle u_{\mathbf{q}} u_{-\mathbf{Q}-\mathbf{q}} u_{-\mathbf{q}_{2}} u_{\mathbf{Q}+\mathbf{q}_{2}}\right\rangle\left[\mathbf{q}_{\perp}\left(\mathbf{Q}+\mathbf{q}_{\perp}\right)\right]\left[\mathbf{q}_{2 \perp}\left(\mathbf{Q}+\mathbf{q}_{2 \perp}\right)\right]
$$

The summation is performed over the 3 directions of the reciprocal space. We observe that $I(\mathbf{Q})$ is strongly non-linear, since it couples all deformation modes.

Only the terms of the form $\left\langle u_{\mathbf{q}} u_{-\mathbf{q}} u_{\mathbf{q}^{\prime}} u_{-\mathbf{q}^{\prime}}\right\rangle$ give non-zero thermal average (bracket). We have 2 cases :

1) $\mathbf{q}_{2}=\mathbf{q}$ which gives terms of the form:

$$
\sum_{\mathbf{q}_{1}}\left\langle\left|u_{\mathbf{q}_{1}}\right|^{2}\right\rangle\left\langle\left|u_{\mathbf{q}+\mathbf{q}_{1}}\right|^{2}\right\rangle
$$

2) $\mathbf{q}_{2}=-\mathbf{Q}-\mathbf{q}$ which gives similar terms

Finally :

$$
I(\mathbf{Q})=\frac{V^{2} c_{0}^{2}}{2} \sum_{\mathbf{q}}\left\langle\left|u_{\mathbf{q}}\right|^{2}\right\rangle\left\langle\left|u_{\mathbf{Q}+\mathbf{q}}\right|^{2}\right\rangle\left[\mathbf{q}_{\perp}\left(\mathbf{Q}+\mathbf{q}_{\perp}\right)\right]^{2}
$$

We have for smectic A phases [8]:

$$
\left\langle\left|u_{\mathbf{q}}\right|^{2}\right\rangle=\frac{k_{\mathrm{B}} T}{V} \frac{1}{K q_{\perp}^{4}+\bar{B} q_{z}^{2}}
$$

$K$ is the smectic rigidity; it is related to the lamella rigidity through $K=k_{\mathrm{c}} / d$.

$\bar{B}$ is the smectic compressibility.

$$
\Rightarrow I(\mathbf{Q})=\frac{\left(k_{\mathrm{B}} T c_{0}\right)^{2}}{2} \sum_{\mathbf{q}} \frac{\left[\mathbf{q}_{\perp}\left(\mathbf{Q}+\mathbf{q}_{\perp}\right)\right]^{2}}{\left(K q_{\perp}^{4}+\bar{B} q_{z}^{2}\right)\left(K\left(\mathbf{Q}+\mathbf{q}_{\perp}\right)^{4}+\bar{B} q_{z}^{2}\right)} .
$$

Replacing the discrete summation by the integral :

$$
\frac{V}{(2 \pi)^{3}} \int_{-\infty}^{+\infty} \int_{-\infty}^{+\infty} \mathrm{d} \mathbf{q}_{\perp} \int_{-\frac{\pi}{d}}^{\frac{\pi}{d}}(\ldots) \mathrm{d} q_{z}
$$

leads to

$$
I(\mathbf{Q})=\frac{\left(k_{\mathrm{B}} T c_{0}\right)^{2} V}{16 \pi^{3}} \int \frac{\left[q_{\perp}^{2}-\frac{Q^{2}}{4}\right]^{2}}{\left(K\left(\mathbf{q}_{\perp}-\frac{\mathbf{Q}}{2}\right)^{4}+\bar{B} q_{z}^{2}\right)\left(K\left(\mathbf{q}_{\perp}+\frac{\mathbf{Q}}{2}\right)^{4}+\bar{B} q_{z}^{2}\right)} \mathrm{d} \mathbf{q} .
$$


The integration over $q_{z}$ gives

$$
\begin{aligned}
& I(\mathbf{Q})=\frac{\left(k_{\mathrm{B}} T \dot{c}_{0}\right)^{2} V}{(2 \pi)^{3} K^{3 / 2} \bar{B}^{1 / 2}} \int \frac{\left[q_{\perp}^{2}-\frac{Q^{2}}{4}\right]^{2}}{\left(\mathbf{q}_{\perp}+\frac{\mathbf{Q}}{2}\right)^{4}-\left(\mathbf{q}_{\perp}-\frac{\mathbf{Q}}{2}\right)^{4}} \times \\
& \times\left[\frac{\tan ^{-1}\left(\frac{\pi}{d} \sqrt{\frac{\bar{B}}{K}} \frac{1}{\left(\mathbf{q}_{\perp}-\frac{\mathbf{Q}}{2}\right)^{2}}\right)}{\left(\mathbf{q}_{\perp}-\frac{\mathbf{Q}}{2}\right)^{2}}-\frac{\tan ^{-1}\left(\frac{\pi}{d} \sqrt{\left.\frac{\bar{B}}{K} \frac{1}{\left(\mathbf{q}_{\perp}+\frac{\mathbf{Q}}{2}\right)^{2}}\right)}\right.}{\left(\mathbf{q}_{\perp}+\frac{\mathbf{Q}}{2}\right)^{2}}\right] \mathrm{d} \mathbf{q}_{\perp} .
\end{aligned}
$$

And after some calculation we finally obtain

$$
I(\mathbf{Q})=\frac{\left(k_{\mathrm{B}} T c_{0}\right)^{2} V}{(2 \pi)^{3} K^{3 / 2} \bar{B}^{1 / 2}} f\left(\frac{\pi}{d} \sqrt{\frac{\bar{B}}{K}} \frac{1}{Q^{2}}\right)
$$

where $f$ is a numerical function

$$
\begin{aligned}
& f(\gamma)=\int_{-\infty}^{+\infty} \int_{-\infty}^{+\infty} \frac{\left(\phi^{2}+\psi^{2}-\frac{1}{4}\right)^{2}}{\left[\left(\phi+\frac{1}{2}\right)^{2}+\psi^{2}\right]^{2}-\left[\left(\phi-\frac{1}{2}\right)^{2}+\psi^{2}\right]^{2}} \times \\
& \times\left[\frac{\tan ^{-1}\left(\frac{\gamma}{\left(\phi-\frac{1}{2}\right)^{2}+\psi^{2}}\right)}{\left(\phi-\frac{1}{2}\right)^{2}+\psi^{2}}-\frac{\tan ^{-1}\left(\frac{\gamma}{\left(\phi+\frac{1}{2}\right)^{2}+\psi^{2}}\right)}{\left(\phi+\frac{1}{2}\right)^{2}+\psi^{2}}\right] \mathrm{d} \phi \mathrm{d} \psi
\end{aligned}
$$

The function $f$ is shown in figure 3 .

Let us now focus on the limiting cases :

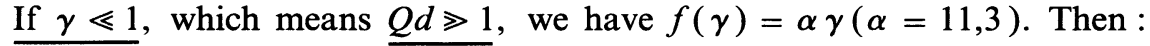

$$
I(Q)=\alpha \frac{\left(k_{\mathrm{B}} T c_{0}\right)^{2} V}{8 \pi^{2} K^{2} Q^{2} d} .
$$

The smectic rigidity is related to the lamella rigidity by $K=k_{\mathrm{c}} / d$. Then, using $c_{0}=\frac{2}{d a^{2}}$ we obtain in the high $Q$ limit :

$$
I(Q)=\alpha \frac{\left(k_{\mathrm{B}} T\right)^{2} V}{2 \pi^{2} a^{4} k_{\mathrm{c}}^{2} d} \cdot \frac{1}{Q^{2}} \quad(Q d \gg 1) .
$$

We note that $I(Q)$ is independent of the interaction parameter $\bar{B}$ and is proportional to the number of lamellae in the sample (i.e. to $d^{-1}$ ), which is indeed expected in the high $Q$ limit, where we expect a free-lamella behaviour. 
(a)

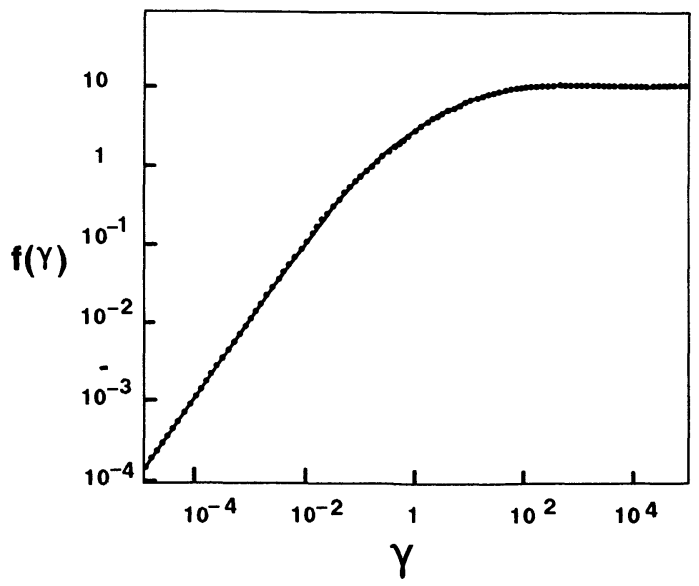

(b)

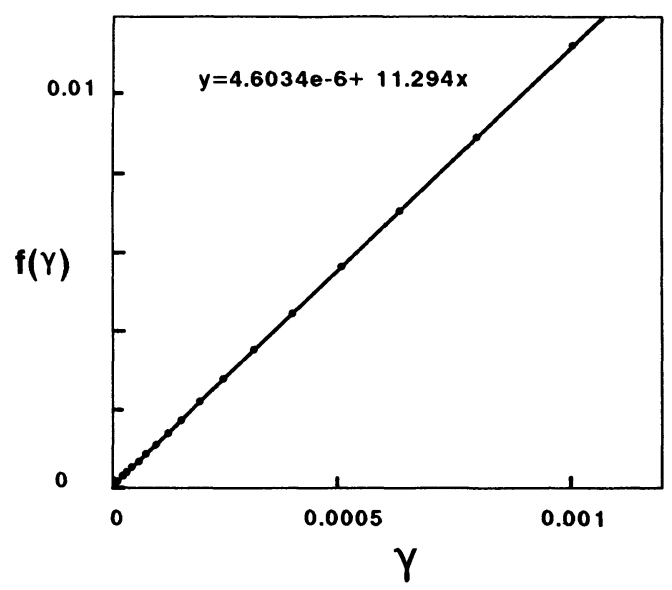

Fig. 3. - Numerical calculation of the static function $f$. a) Whole range. At high $\gamma$ (small $Q) f$ is constant $f(\infty)=11,2$. b) Detail of the small $\gamma$ area. We see that $f$ is perfectly linear and $f(\gamma)=11.3 \gamma$.

If $\gamma \gg 1$, then $Q d \ll 1, f$ is a constant $\beta=11,2:$ the $\tan ^{-1}$ in the integral is constant, except at great values of $\phi$ and $\psi$, but then the other factors are very small and the integrant is almost zero.

We then have in the low $Q$ limit:

$$
I(Q)=4 \beta \frac{\left(k_{\mathrm{B}} T\right)^{2} V}{(2 \pi)^{3} a^{4} K^{3 / 2} \bar{B}^{1 / 2} d^{2}} \quad(Q d \ll 1)
$$

$I(Q)$ is therefore a constant in the low- $Q$ limit. For the sake of comparison with the predictions obtained in references [12], we interprete formally $I(Q)$ in this range in terms of an equivalent compressibility $\chi$ :

$$
I(Q \rightarrow 0)=\frac{k_{\mathrm{B}} T}{V} \chi N^{2}
$$

where $N$ is the number of surfactant molecules in the sample : $N=\frac{2 V}{e a^{2}}(e$ is the thickness of the bilayer).

Equation (11) then gives :

$$
\chi=\frac{\beta k_{\mathrm{B}} T \mathrm{e}^{2}}{(2 \pi)^{3} d^{2} K^{3 / 2} \bar{B}^{1 / 2}}
$$

using $K=k_{\mathrm{c}} / d$ and replacing $\bar{B}$ by its value in the case of purely steric interaction [3] :

$$
\bar{B}_{\mathrm{st}} \sim \frac{\left(k_{\mathrm{B}} T\right)^{2}}{k_{\mathrm{c}} d^{3}}
$$


we obtain :

$$
\chi \sim \frac{d e^{2}}{k_{\mathrm{c}}}
$$

which agrees with reference [12].

A useful notation for $I(Q)$ may be, using a normalization by $I(0)$ :

$$
\frac{I\left(Q \gg d^{-1}\right)}{I(0)}=\frac{\pi}{d} \sqrt{\frac{\bar{B}}{K}} \frac{1}{Q^{2}}
$$

This formula allows the use of relative intensity measurements.

\section{Dynamic neutron scattering.}

The dynamic intensity is, similarly :

$$
\begin{aligned}
F(\mathbf{Q}, t) & =\left\langle\int \delta c\left(\mathbf{r}_{1}, t\right) \mathrm{e}^{i \mathbf{Q} \cdot \mathbf{r}_{1}} \mathrm{~d} \mathbf{r}_{1} \int \delta c^{*}\left(\mathbf{r}_{2}, 0\right) \mathrm{e}^{-i \mathbf{Q} \cdot \mathbf{r}_{2}} \mathrm{dr}_{2}\right\rangle \\
& =\frac{V^{2} c_{0}^{2}}{4} \sum_{\mathbf{q}, \mathbf{q}_{2}}\left\langle u_{\mathbf{q}}(t) u_{-\mathbf{Q}-\mathbf{q}}(t) u_{-\mathbf{q}_{2}}(0) u_{\mathbf{Q}+\mathbf{q}_{2}}(0)\right\rangle\left[\mathbf{q}_{\perp}\left(\mathbf{Q}+\mathbf{q}_{\perp}\right)\right]\left[\mathbf{q}_{2 \perp}\left(\mathbf{Q}+\mathbf{q}_{2 \perp}\right)\right] .
\end{aligned}
$$

Following preceding calculations :

$$
F(\mathbf{Q}, t)=\frac{V^{2} c_{0}^{2}}{4} \sum_{\mathbf{q}}\left\langle u_{\mathbf{q}}(t) u_{-\mathbf{q}}(0)\right\rangle\left\langle u_{\mathbf{Q}+\mathbf{q}}(t) u_{-\mathbf{Q}-\mathbf{q}}(0)\right\rangle\left[\mathbf{q}_{\perp}\left(\mathbf{Q}+\mathbf{q}_{\perp}\right)\right]^{2}
$$

And :

$$
\left\langle u_{\mathbf{q}}(t) u_{-\mathbf{q}}(0)\right\rangle=\left\langle\left|u_{\mathbf{q}}\right|^{2}\right\rangle \mathrm{e}^{-\frac{t}{\tau(\mathbf{q})}}
$$

where $\tau(\mathbf{q})$ is the hydrodynamic relaxation time. Then :

$$
F(\mathbf{Q}, t)=\frac{\left(k_{\mathrm{B}} T c_{0}\right)^{2} V}{16 \pi^{3}} \int \frac{\left[q_{\perp}^{2}-\frac{Q^{2}}{4}\right]^{2}}{\left(K\left(\mathbf{q}_{\perp}-\frac{\mathbf{Q}}{2}\right)^{4}+\bar{B} q_{z}^{2}\right)\left(K\left(\mathbf{q}_{\perp}+\frac{\mathbf{Q}}{2}\right)^{4}+\bar{B} q_{z}^{2}\right)} \mathrm{e}^{-\frac{t}{\tau(\mathbf{q})}} \mathrm{d} \mathbf{q} .
$$

Since $\mathbf{q}$ is generally oblique, we do not know the general form of $\tau(\mathbf{q})$. Only the case $Q d \gg 1$ may be explicited.

In this case, we see in equation (17) that the numerator is of the order of $Q^{4}$, while the denominator is of the order of $Q^{8}$. The integrant is then generally small, except in the following cases :

$$
\mathbf{q}_{\perp} \approx \frac{\mathbf{Q}}{2} \quad \text { or } \quad \mathbf{q}_{\perp} \approx-\frac{\mathbf{Q}}{2}
$$

But in these cases, since $\left|q_{z}\right| \leq \frac{\pi}{d}, q_{z}$ is very small in comparison with $q_{\perp}$ so $\mathbf{q}$ is almost 
parallel to the lamella. We shall then use the relaxation time calculated for undulation modes, which is, in the high- $q$ limit :

$$
\tau(\mathbf{q})=\frac{4 \eta}{k_{\mathrm{c}} q^{3}}
$$

Equation (17) then becomes :

$$
F(\mathbf{Q}, t)=\frac{\left(k_{\mathrm{B}} T c_{0}\right)^{2} V}{(2 \pi)^{3} K^{3 / 2} \bar{B}^{1 / 2}} g\left(\frac{\pi}{d} \sqrt{\frac{\bar{B}}{K}} \frac{1}{Q^{2}}, \frac{t}{\tau(Q)}\right) \quad(Q d \gg 1)
$$

where :

$$
\begin{aligned}
g(\gamma, \lambda)=\int_{-\infty}^{+\infty} \int_{-\infty}^{+\infty} \frac{\left(\phi^{2}+\psi^{2}-\frac{1}{4}\right)^{2}}{\left[\left(\phi+\frac{1}{2}\right)^{2}+\psi^{2}\right]^{2}-\left[\left(\phi-\frac{1}{2}\right)^{2}+\psi^{2}\right]^{2}} \times \\
\times\left[\frac{\tan ^{-1}\left(\frac{\gamma}{\left(\phi-\frac{1}{2}\right)^{2}+\psi^{2}}\right)}{\tan ^{-1}\left(\frac{\gamma}{\left(\phi+\frac{1}{2}\right)^{2}+\psi^{2}}\right)}\right) \\
\times \mathrm{e}^{-\lambda\left[\left(\left(\phi-\frac{1}{2}\right)^{2}+\psi^{2}\right)^{3 / 2}+\left(\left(\phi+\frac{1}{2}\right)^{2}+\psi^{2}\right)^{3 / 2}\right]} \mathrm{d} \phi \mathrm{d} \psi .
\end{aligned}
$$

Now, let us restrict the integration at $\phi, \psi>0$. As the integrand is symmetric on $\phi$ and $\psi, g$ is divided by 4 .

We can now see that, since $\gamma$ is small, the second $\tan ^{-1}$ is always small, while the first takes significant values only if $\phi \rightarrow 1 / 2$ and $\psi \rightarrow 0$. The $\tan ^{-1}$ then works as a $\delta$ function, and we replace $\phi$ and $\psi$ by these values in the exponential. We then have :

$$
g(\gamma, \lambda)=\mathrm{e}^{-\lambda} f(\gamma) .
$$

Then in the high $Q$ limit we have :

$$
F(Q, t)=I(Q) \mathrm{e}^{-\frac{1}{\tau}}
$$$$
\begin{aligned}
& \text { with } \tau=\frac{4 \eta}{k_{\mathrm{c}} Q^{3}} \\
& \text { and } \quad Q d \gg 1
\end{aligned}
$$

The dynamic intensity is then exponential and gives immediately $\tau$, and then $k_{\mathrm{c}}$.

\section{Conclusion.}

We have calculated the relaxation time of elastic displacement modes with the wave vector $\mathbf{q}$ parallel to the bilayers for lyotropic lamellar phases. The local formalism used here allows us to calculate this time over the entire accessible $q$ range. The general problem of oblique $q$ involves the interaction between the bilayers and it is still unsolved for high $q$ values in swollen lamellar phases stabilized by the steric interaction : the basic difficulty being that the 
harmonic approximation $(\bar{B})$ for this interaction is certainly not valid for high $q_{\perp}$ and $q_{z} \approx \pi / d$.

Secondly, we have calculated the static intensity scattered by these phases for scattering wave vector $\mathbf{Q}$ parallel to the layers. We obtain a $Q^{-2}$ dependence at high $Q$ and a constant at small $Q$.

Finally, the dynamic intensity has also been calculated in the high $Q$ regime : it is monoexponential and defines a relaxation time $\tau$ which is the one calculated in the hydrodynamic theory for the pure undulation mode of wave vector $\mathbf{q}=\mathbf{Q}$.

\section{References}

[1] Ekwall P., Advances in liquid crystals, Ed. G. M. Brown (Academic Press) 1975.

[2] Benton W. J. and Miller C. A., J. Phys. Chem. 87 (1983) 4981 ;

HARbich W. and Helfrich W., Chem.Phys. Lipids 36 (1984) 39 ;

di Meglio J. M., Dvolaitzky M. and Taupin C., J. Phys. Chem. 89 (1985) 871 ;

Larché F. C., Marignan J. and el Quebraj S., J. Phys. Chem. 90 (1986) 707.

[3] Helfrich W., Z. Natürforsch. A 33 (1978) 305.

[4] Caille A., C. R. Heb. Sean. Acad. Sci. B 274 (1972) 891 ;

Gunther L., IMry Y. and Lajzerowicz J., Phys. Rev. A 22 (1980) 1733 ;

Larché F. C., Appell J., Porte G., Bassereau P. and Marignan J., Phys. Rev. Lett. 56 (1986) 1700 ;

Safinya C. R., Roux D., Smith G. S., Sinha S. K., Dimon P., Clark N. A. and Belloce A. M., Phys. Rev. Lett. 57 (1986) 2518 ;

Bassereau P., Marignan J. and Porte G., J. Phys. France 48 (1987) 673 ;

Roux D. and SAFinYa C. R., J. Phys. France 49 (1988) 117.

[5] Porte G., Marignan J., Bassereau P. and May R., Europhys. Lett. 7 (1988) 713.

[6] Nallet F., Roux D. and Prost J., J. Phys. France 50 (1989) 3147.

[7] Landau L. and Lifchitz E., Statistical Physics (Mir, Moscou) 1976.

[8] De Gennes P. G., The Physics of Liquid Crystals (Clarendon Press, Oxford) 1974.

[9] Brochard F. and Lennon J. F., J. Phys. 11 (1975) 1035.

[10] Milner S. T. and Safran S. A., Phys. Rev. A 36 (1987) 4371.

[11] LOVESEY S. W., Theory of neutron scattering from condensed matter (Clarendon Press, Oxford) 1984.

[12] Lubensky T. C., Prost J. and Ramaswamy S., preprint. 